\title{
Rhabdomyolysis in COVID-19 Patients: A Retrospective Observational Study
}

Magued W. Haroun ${ }^{1}$, Vladyslav Dieiev ${ }^{1}$, John Kang ${ }^{1}$, Mali Barbi ${ }^{1}$, Seyed Farzad Marashi Nia ${ }^{1}$, Mohamed Gabr $^{1}$, Gerardo Eman ${ }^{1}$, Grace Kajita ${ }^{1}$, Kristin Swedish ${ }^{1}$

1. Internal Medicine, Montefiore Medical Center, Wakefield Campus, Bronx, USA

Corresponding author: Magued W. Haroun, mharoun@montefiore.org

\section{Abstract}

\section{Background}

The coronavirus disease 2019 (COVID-19) pandemic has caused significant morbidity and mortality worldwide. Knowledge about the pathophysiology of the disease and its effect on multiple systems is growing. Kidney injury has been a topic of focus, and rhabdomyolysis is suspected to be one of the contributing mechanisms. However, information on rhabdomyolysis in patients affected by COVID-19 is limited. We aim to describe the incidence, clinical characteristics, and outcomes of patients hospitalized with COVID-19 who developed rhabdomyolysis.

\section{Materials and methods}

A retrospective observational cohort consisted of patients who were admitted and had an outcome between March 16 to May 27, 2020, inclusive of those dates at a single center in the Bronx, New York City. All consecutive inpatients with lab-confirmed COVID-19 were identified. Patients with peak total creatine kinase (CK) over 1,000 U/L were reviewed; 140 patients were included in the study. The main outcomes during hospitalization were new-onset renal replacement therapy and in-hospital mortality.

\section{Results}

The median age was 68 years (range: $21-93) ; 64 \%$ were males. The most common comorbidities were hypertension (73\%), diabetes mellitus (47\%), and chronic kidney disease (24\%). Median CK on admission was $1,323 \mathrm{U} / \mathrm{L}$ (interquartile range [IQR]: 775 - 2,848). Median CK on discharge among survivors was 852 (IQR: 170 - 1,788). Median creatinine on admission was $1.78 \mathrm{mg} / \mathrm{dL}$ (IQR: 1.23 - 3.06). During hospitalization, 49 patients (35\%) received invasive mechanical ventilation, 24 patients $(17.1 \%)$ were treated with renal replacement therapy (RRT), and 66 (47.1\%) died.

\section{Conclusions}

Rhabdomyolysis was a common finding among hospitalized patients with COVID-19 in our hospital in the Bronx. The incidence of new-onset renal replacement therapy and in-hospital mortality is higher in patients who develop rhabdomyolysis. McMahon score, rather than isolated creatine kinase levels, was a statistically significant predictor of new-onset RRT. Clinicians should maintain a high level of suspicion for

Review began 12/14/2020 Review ended 01/04/202 Published 01/07/2021

\section{() Copyright 2021}

Haroun et al. This is an open access article distributed under the terms of the Creative Commons Attribution License CC-BY 4.0., which permits unrestricted use, distribution, and reproduction in any medium, provided the original author and source are credited. rhabdomyolysis in COVID-19 patients throughout their admission and use validated scores like McMahon score to devise their treatment plan accordingly.

\section{Categories: Internal Medicine, Infectious Disease, Nephrology}

Keywords: covid-19, acute kidney injury, rhabdomyolysis, hemodialysis, muscle breakdown

\section{Introduction}

Rhabdomyolysis is a syndrome characterized by skeletal muscle breakdown with the resultant release of intracellular contents into the bloodstream leading to systemic complications, most commonly acute kidney injury (AKI). Creatine kinase (CK) is the most sensitive indicator of muscle damage and has been used for laboratory confirmation of rhabdomyolysis [1]. It has been widely accepted that CK levels more than five times the upper limit of normal $(\geqslant 1,000 \mathrm{U} / \mathrm{L})$ are diagnostic of rhabdomyolysis.

There are multiple causes of rhabdomyolysis. Viral infections causing rhabdomyolysis are more common in the pediatric population but have been reported frequently also in adults. Many viral, bacterial, fungal, and protozoal infections have been associated with rhabdomyolysis, of which influenza A is the most commonly implicated [2]. More recently, rhabdomyolysis has been reported as a complication of the severe acute respiratory syndrome (SARS) caused by SARS-CoV [3]. The exact mechanism of muscle damage in viral infections has not been established.

The world is currently facing a global health crisis caused by severe acute respiratory syndrome coronavirus 
2 (SARS-CoV-2). Understanding of the full spectrum of coronavirus disease 2019 (COVID-19), the disease caused by the new virus, is evolving. Since the beginning of the pandemic, it was noted that CK elevation above the upper limit of normal (>185 U/L) correlated with severe disease and increased mortality [4]. In one study, serum CK decline correlated with viral mRNA elimination [5]. However, rhabdomyolysis in COVID-19 patients has not been commonly reported - a few retrospective cohorts reported peak CK over 1,000 U/L and to date, few case reports have described patients who developed severe rhabdomyolysis (peak CK of approximately 10,000 U/L) during the course of illness [6-10].

Kidney involvement is common in COVID-19. The spectrum ranges from mild proteinuria to progressive AKI requiring renal replacement therapy (RRT) [11]. The pathophysiology of AKI is likely multifactorial and is under active investigation. Rhabdomyolysis is suspected to be one of the contributors to AKI in COVID-19 patients. In a post-mortem renal histopathological analysis of COVID-19 patients in China, pigmented casts were found with high CK levels in some cases, possibly representing rhabdomyolysis [12].

A better understanding of the incidence of rhabdomyolysis in COVID-19 patients and its clinical implications is important to fully appreciate the spectrum of damage caused by the virus and guide decision making regarding fluid management and prognostication. Our study aims to shed light on the burden of rhabdomyolysis in COVID-19 patients and to describe the demographic and clinical characteristics, laboratory findings, and outcomes among patients who presented with or developed this condition during their admission in a hospital at one of the hardest hit boroughs of New York City.

\section{Materials And Methods}

This is a retrospective observational cohort study. We obtained data from a teaching hospital (Montefiore Medical Center [MMC] - Wakefield campus) in the Bronx borough of New York City on hospitalized patients and had an outcome between March 16 and May 27, 2020. Patients were found by applying search criteria to the hospital's electronic medical records (EMR) registry. We then obtained information by manual chart review on patient demographics (age, gender, body-mass index [BMI], and race/ethnicity), presenting symptoms, vital status at hospital discharge, electronic discharge summaries, and inpatient laboratory values. Approval for this study was granted by the institutional review board at Albert Einstein College of Medicine; the need for informed consent was waived because of the retrospective nature of the study.

Inclusion criteria were age older than 18 years, lab-confirmed COVID-19 (positive result for SARS-CoV-2 in reverse transcriptase-polymerase chain reaction assay of nasal or nasopharyngeal swabs), and peak total CK level more than 1,000 U/L during hospitalization. We excluded patients with end-stage renal disease (ESRD) and those who had evidence of a secondary cause of rhabdomyolysis, including seizures, acute coronary syndrome or crush injuries prior to admission. Primary outcomes were in-hospital mortality and new-onset RRT. The only RRT modality offered in our campus was intermittent hemodialysis.

AKI was defined by Kidney Disease Improving Global Outcomes (KDIGO) criteria [13]. Baseline creatinine was determined by reference to preadmission creatinine value within six months of admission or, if not available, the lowest creatinine measured during hospitalization. McMahon score was calculated for every patient on admission. It includes eight variables: age, gender, etiology, and initial levels of CK, creatinine, calcium, phosphate, and serum bicarbonate. McMahon score has been validated as an instrument for predicting mortality and AKI. A score greater than six is $86 \%$ sensitive and $68 \%$ specific for identifying patients who will require RRT [14-15].

Eight-hundred twenty-five patients were admitted to MMC Wakefield hospital during the study period with laboratory-confirmed COVID-19. One-hundred sixty-five patients (17\%) met the criteria for rhabdomyolysis diagnosis ( $\mathrm{CK}>1,000 \mathrm{U} / \mathrm{L}$ ) during their admission. Twenty-five patients were excluded based on exclusion criteria mentioned prior; 140 patients were included in the study.

This research was done without patient involvement. Patients were not invited to comment on the study design and were not consulted to develop patient-relevant outcomes or interpret the results. Patients were not invited to contribute to the writing or editing of this document for readability or accuracy.

Descriptive statistics were used to summarize the data; results are reported as medians and interquartile ranges (IQR) or means and standard deviations, as appropriate. Categorical variables were summarized as counts and percentages. Univariate logistic regression was used to assess the effect of demographics, comorbidities, laboratory data, and invasive mechanical ventilation on mortality and new-onset RRT; multivariate models were created including all variables with univariate $p$-values $<0.2$. All statistical analyses were performed using SPSS statistics software (IBM Inc., Armonk, USA). No imputation was made for missing data.

\section{Results}

\section{Demographic and clinical characteristics}

Demographics and clinical characteristics of our patients are shown in Table 1 . The median age was 68 years 


\section{Cureus}

(range: 21-93); 64\% were men. The most common presenting symptoms were shortness of breath (62\%), fever (50\%), and cough (49\%). The most common chronic medical conditions in this population were hypertension (72\%), diabetes mellitus (47\%), and chronic kidney disease (24\%). Forty-one patients (29\%) were taking statins prior to admission. Forty-nine patients (35\%) received treatment in the intensive care unit for at least 24 hours. 


\section{Cureus}

Characteristic

Median age (range) - yr.

Gender - no. (\%)

Male

Female

BMI - median (IQR)

Race and ethnicity - no. (\%)

African American

Hispanic

White

Asian

Missing or other

Coexisting disorder - no. (\%)

Hypertension

Diabetes mellitus

Chronic kidney disease

Dementia

Asthma

Hemorrhagic or ischemic stroke

Chronic obstructive pulmonary disease

Atrial fibrillation

Coronary artery disease

Seizure disorder

Statin use prior to admission - no. (\%)

Respiratory symptoms - no. (\%)

Shortness of breath

Cough

Systemic symptoms - no. (\%)

Fever

Altered mental status

Diarrhea

Weakness

Myalgias

Chest pain
Patients ( $\mathbf{N}=131)$

$68(21-93)$

90 (64.3)

50 (35.7)

$29.5(25.2-35.1)$

$85(60.7)$

$29(20.7)$

7 (5)

$1(0.7)$

18 (12.9)

101 (72.1)

65 (46.4)

34 (24.3)

22 (15.7)

17 (12.1)

$13(9.3)$

12 (8.6)

7 (5)

7 (5)

5 (3.6)

41 (29.3)

$87(62.1)$

68 (48.6)

$70(50)$

26 (18.6)

16 (11.4)

18 (12.9)

12 (8.6)

$10(7.1)$

TABLE 1: Clinical characteristics of the patients at baseline

\section{Laboratory results}

Laboratory data during hospitalization are summarized in Table 2. The total CK lab assay at our institution has a maximum limit of $80,000 \mathrm{U} / \mathrm{L}$. Results greater than $80,000 \mathrm{U} / \mathrm{L}$ are reported as $(>80,000 \mathrm{U} / \mathrm{L})$. Median 


\section{Cureus}

CK on admission was 1,323 U/L (IQR: 775 - 2,848). Median CK on discharge among survivors was 852 (IQR: 170 - 1,788). Median creatinine on admission was $1.78 \mathrm{mg} / \mathrm{dL}$ (IQR: 1.23 - 3.06).

\begin{tabular}{|c|c|c|}
\hline Laboratory Data & Patients (N=140) & Reference range \\
\hline \multicolumn{3}{|l|}{ Creatine kinase $-U / L$} \\
\hline Admission, median (IQR) & $1,323(775-2,848)$ & $<200 \mathrm{U} / \mathrm{L}$ \\
\hline Peak, median (IQR) & $2,209(1,283-4,161)$ & $<200$ U/L \\
\hline \multicolumn{3}{|l|}{ Creatinine $-\mathrm{mg} / \mathrm{dL}$} \\
\hline Admission, median (IQR) & $1.78(1.23-3.06)$ & $<1.20 \mathrm{mg} / \mathrm{dL}$ \\
\hline Peak, median (IQR) & $3.02(1.60-6.48)$ & $<1.20 \mathrm{mg} / \mathrm{dL}$ \\
\hline \multicolumn{3}{|l|}{ C-Reactive Protein - mg/dL } \\
\hline Peak, median (IQR) & $21.2(10.9-33.1)$ & $<0.8 \mathrm{mg} / \mathrm{dL}$ \\
\hline \multicolumn{3}{|l|}{ D-Dimer - ug/mL FEU } \\
\hline Peak, median (IQR) & $6.88(2.29-20)$ & $0.00-0.05 \mathrm{ug} / \mathrm{mL}$ FEU \\
\hline \multicolumn{3}{|c|}{ McMahon's score on admission } \\
\hline$<6$, no. $(\%)$ & $31(22.1)$ & \\
\hline$\geq 6$, no. $(\%)$ & $109(77.9)$ & \\
\hline
\end{tabular}

\section{TABLE 2: Laboratory data during hospitalization}

IQR - interquartile range; FEU - fibrinogen equivalent units

\section{Outcomes}

Out of the 140 patients who were included in this study, 74 (52.9\%) were discharged, and 66 (47.1\%) died in the hospital. One-hundred-and-twelve patients (80\%) developed AKI, twenty-four (17.1\%) of which required RRT. Five patients were planned for RRT but expired prior to RRT initiation or family refused RRT. All patients planned for RRT were included in the final analysis. Results of our univariate and multivariable logistic regression model for mortality and new-onset RRT are summarized in Tables 3 and 4, respectively. 


\section{Cureus}

\begin{tabular}{|c|c|c|c|c|}
\hline & Univariate OR (95\% Cl) & $\mathrm{p}$-value & Multivariate OR $(95 \% \mathrm{Cl})$ & p-value \\
\hline \multicolumn{5}{|l|}{ Demographics } \\
\hline Age & $1.05(1.02-1.08)$ & 0.000 & $1.06(1.01-1.12)$ & 0.027 \\
\hline Male gender & $1.35(0.68-2.71)$ & 0.391 & & \\
\hline BMI & $0.99(0.96-1.04)$ & 0.968 & & \\
\hline \multicolumn{5}{|l|}{ Co-morbidities } \\
\hline Hypertension & $2.21(1.02-4.78)$ & 0.044 & & \\
\hline Diabetes mellitus & $1.82(0.93-3.57)$ & 0.082 & & \\
\hline Chronic kidney disease & $1.41(0.64-3.07)$ & 0.393 & & \\
\hline Dementia & $2.82(1.07-7.41)$ & 0.036 & $7.72(1.34-44.46)$ & 0.022 \\
\hline \multicolumn{5}{|l|}{ Laboratory measures } \\
\hline \multicolumn{5}{|l|}{ Creatine kinase } \\
\hline Admission & $1.00(1.00-1.00)$ & 0.109 & & \\
\hline Peak & $1.00(1.00-1.00)$ & 0.594 & & \\
\hline Time to peak & $1.07(0.96-1.17)$ & 0.194 & & \\
\hline \multicolumn{5}{|l|}{ Creatinine } \\
\hline Admission & $1.04(0.92-1.18)$ & 0.520 & & \\
\hline Peak & $1.13(1.03-1.24)$ & 0.005 & & \\
\hline C-Reactive protein, peak & $1.06(1.03-1.20)$ & 0.000 & $1.06(1.01-1.11)$ & 0.011 \\
\hline D-Dimer, peak & $1.12(1.05-1.18)$ & 0.000 & & \\
\hline McMahon's score $\geq 6$ & $2.68(1.13-6.34)$ & 0.025 & & \\
\hline \multicolumn{5}{|l|}{ Clinical measures } \\
\hline AKI & $2.69(1.09-6.60)$ & 0.031 & & \\
\hline New-onset RRT & $2.12(0.86-5.25)$ & 0.102 & $3.77(0.83-17.13)$ & 0.085 \\
\hline Invasive mechanical ventilation & $8.69(3.80-19.84)$ & 0.000 & $16.34(4.43-60.23)$ & 0.000 \\
\hline
\end{tabular}

\section{TABLE 3: Predictors of mortality}

OR - odds ratio; $\mathrm{Cl}$ - confidence interval; BMI - body mass index; AKI - acute kidney injury; RRT - renal replacement therapy 


\section{Cureus}

\begin{tabular}{|c|c|c|c|c|}
\hline & Univariate OR (95\% Cl) & $\mathrm{p}$-value & Multivariate OR $(95 \% \mathrm{Cl})$ & p-value \\
\hline \multicolumn{5}{|l|}{ Demographics } \\
\hline Age & $0.98(0.95-1.01)$ & 0.161 & $0.893(0.838-0.951)$ & 0.000 \\
\hline Male gender & $0.63(0.25-1.54)$ & 0.308 & & \\
\hline BMI & $1.04(0.99-1.09)$ & 0.099 & & \\
\hline \multicolumn{5}{|l|}{ Co-morbidities } \\
\hline Hypertension & $2.88(0.93-8.90)$ & 0.066 & $64.84(2.72-1548.14)$ & 0.010 \\
\hline Diabetes mellitus & $6.20(2.34-16.49)$ & 0.000 & $5.32(1.07-26.51)$ & 0.041 \\
\hline Chronic kidney disease & $1.74(0.69-4.37)$ & 0.242 & & \\
\hline Dementia & $0.34(0.07-1.53)$ & 0.160 & & \\
\hline \multicolumn{5}{|l|}{ Laboratory measures } \\
\hline \multicolumn{5}{|l|}{ Creatine kinase } \\
\hline Admission & $1.00(0.99-1.00)$ & 0.100 & $0.99(0.99-1.00)$ & 0.058 \\
\hline Peak & $1.00(1.00-1.00)$ & 0.620 & & \\
\hline Time to peak & $1.17(1.05-1.29)$ & 0.003 & & \\
\hline \multicolumn{5}{|l|}{ Creatinine $^{\star \star}$} \\
\hline Admission & $1.37(1.16-1.62)$ & 0.000 & & \\
\hline Peak & $2.40(1.70-3.38)$ & 0.000 & & \\
\hline C-Reactive protein, peak & $1.04(1.01-1.07)$ & 0.007 & & \\
\hline D-Dimer, peak & $1.05(0.99-1.11)$ & 0.100 & & \\
\hline \multicolumn{5}{|l|}{ Clinical measures } \\
\hline McMahon's score $\geq 6$ & $10.37(1.35-79.61)$ & 0.024 & $100.21(4.53-2215.1)$ & 0.004 \\
\hline Invasive mechanical ventilation & $5.37(2.24-12.89)$ & 0.000 & & \\
\hline
\end{tabular}

\section{TABLE 4: Predictors of new-onset RRT*}

*Including patients who were planned for RRT but expired prior to the actual initiation of RRT.

${ }^{\star \star}$ Creatinine values were not included in this multivariate model to avoid confounding.

OR - odds ratio; Cl - confidence interval; BMI - body mass index; RRT - renal replacement therapy

Outcomes as compared to MMC Wakefield COVID-19 admissions who had peak CK less than 1,000 U/L during the same period are summarized in Table 5. The incidence of rhabdomyolysis among all COVID-19 patients admitted to our hospital during the study period was $16.9 \%$. The in-hospital mortality rate in COVID-19 patients who developed rhabdomyolysis was significantly higher than mortality among COVID-19 patients who did not develop rhabdomyolysis (47.1\% vs. $26.4 \%$ ). The incidence of new-onset RRT among COVID-19 patients who developed rhabdomyolysis was over triple the incidence in COVID-19 patients who did not develop rhabdomyolysis (17.1 vs. $2.9 \%$ ). 


\section{Cureus}

\begin{tabular}{|l|l|l|}
\hline & $\begin{array}{l}\text { Patients admitted with COVID-19 and peak CK } \\
<1000 \mathrm{U} / \mathrm{L}\end{array}$ & $\begin{array}{l}\text { Study group: patients admitted with COVID-19 and } \\
\text { peak CK }>1000\end{array}$ \\
\hline $\mathrm{N}$ & 685 & 140 \\
\hline $\begin{array}{l}\text { New-onset RRT - no. } \\
\text { (\%) }\end{array}$ & $20(2.9)$ & $24(17.1)$ \\
\hline $\begin{array}{l}\text { In-hospital mortality }- \\
\text { no. (\%) }\end{array}$ & $181(26.4)$ & $645(47.1)$ \\
\hline
\end{tabular}

\section{TABLE 5: Outcomes as compared to all COVID-19 admissions during the same time period}

CK - creatine kinase; RRT - renal replacement therapy; COVID-19 - coronavirus disease 2019

Most patients ( $\mathrm{n}=132,94.3 \%$ ) in our cohort met the definition of rhabdomyolysis (CK greater than 1,000 U/L) within the first week of admission, while seven patients developed rhabdomyolysis during their second week of admission. Notably, all seven patients were intubated. In total, 49 patients (35\%) were intubated and mechanically ventilated. The mortality rate among intubated patients was $79.2 \%$.

\section{Discussion}

This study represents the first observational study providing a snapshot of the burden of rhabdomyolysis among COVID-19 patients admitted to one hospital in one of the most affected boroughs at the pandemic epicenter.

Patients who developed rhabdomyolysis had higher rates of mortality and new-onset RRT as compared to COVID-19 patients with peak CK less than 1,000 U/L. However, based on our logistic regression models, CK levels on admission and peak CK levels were not found to be statistically significant predictors of either new-onset RRT or mortality in our study group. McMahon's score on admission had the strongest correlation with new-onset RRT, highlighting its prognostic value in COVID-19 patients with rhabdomyolysis as well as its role in guiding the initiation of renal protective therapies. Other statistically significant predictors of new-onset RRT were young age, diabetes mellitus, hypertension, and admission creatinine. We suspect a young age was a significant predictor because younger patients were more likely to receive more aggressive treatment modalities, including RRT.

In our multivariate model for mortality, age, dementia, peak C-reactive protein (CRP) levels, and mechanical ventilation were found to be statistically significant predictors of mortality. These findings support recent reports correlating age, CRP levels, and dementia with mortality [16-17]. CRP levels and mechanical ventilation likely reflect critical illness leading to higher mortality.

The mechanism of muscle damage in viral infections, particularly COVID-19, is not fully understood. Proposed hypotheses include direct and indirect mechanisms. The first theory is via direct viral muscle invasion. Angiotensin-converting enzyme 2 (ACE2), which was identified as the functional receptor for SARS-CoV and SARS-CoV-2, is present in skeletal muscles [18-19]. SARS-CoV was not detected in skeletal muscle by postmortem examination, and to this date, it is unclear if SARS-CoV-2 infects muscles directly [20]. The second theory postulates that skeletal muscle damage could be caused by the host "cytokine storm"-like immune response [2].

Serum CK usually begins to rise approximately two to 12 hours after onset of muscle injury, peaks within 24 to 72 hours, and then declines gradually over 7-10 days unless there is continuing muscle injury or development of compartment syndrome [1]. While CK is widely used for laboratory diagnosis of rhabdomyolysis, kidney injury is believed to be caused by myoglobin, which peaks and drops much faster than CK. In two published clinical vignettes, COVID-19 patients who developed rhabdomyolysis were reported on admission in one case and as a late complication (on day nine of hospitalization) in another [89]. Most patients (94\%) in our cohort had peak CK on the presentation or within one week of admission, suggesting the onset of muscle injury likely took place around the time they were admitted to the hospital. Only $5 \%$ of patients in our cohort developed late rhabdomyolysis during admission.

Our study had several limitations. First, all data were retrospectively obtained from electronic medical records, so some demographic and clinical data were missing. Second, we were unable to retrieve data pertaining to treatments given to the patients, especially the rate and timing of intravenous hydration. Obtaining accurate fluids data retrospectively would be challenging. Third, the only RRT modality offered in our campus was intermittent hemodialysis, limiting access for hemodynamically unstable patients. Fourth, resource allocation during a time of crisis like this pandemic might have resulted in a lack of hemodialysis 
treatment or shortened hemodialysis time, which could have impacted mortality rates. Fifth, for some predictors of outcomes in our models, the confidence interval was very wide, likely secondary to small sample size. Larger studies are warranted.

\section{Conclusions}

In conclusion, rhabdomyolysis is an important aspect of the multifaceted nature of COVID-19 and is likely underreported. CK levels in isolation might not correlate with outcomes, so clinicians are encouraged to use validated scores like McMahon score to better predict outcomes and guide treatment decisions for patients who develop rhabdomyolysis, most importantly their fluid management, which can be challenging in patients with impending respiratory failure and should be decided on a case-by-case basis.

\section{Additional Information \\ Disclosures}

Human subjects: Consent was obtained by all participants in this study. Albert Einstein College of Medicine issued approval 2020-11532. The need for informed consent was waived because of the retrospective nature of the study. Animal subjects: All authors have confirmed that this study did not involve animal subjects or tissue. Conflicts of interest: In compliance with the ICMJE uniform disclosure form, all authors declare the following: Payment/services info: All authors have declared that no financial support was received from any organization for the submitted work. Financial relationships: All authors have declared that they have no financial relationships at present or within the previous three years with any organizations that might have an interest in the submitted work. Other relationships: All authors have declared that there are no other relationships or activities that could appear to have influenced the submitted work.

\section{References}

1. Keltz E, Khan FY, Mann G: Rhabdomyolysis. The role of diagnostic and prognostic factors . Muscles Ligaments Tendons J. 2013, 3:303-312.

2. Fadila MF, Wool KJ: Rhabdomyolysis secondary to influenza a infection: a case report and review of the literature. N Am J Med Sci. 2015, 7:122-124. 10.4103/1947-2714.153926

3. Chen L-L, Hsu C-W, Tian Y-C, Fang J-T: Rhabdomyolysis associated with acute renal failure in patients with severe acute respiratory syndrome. Int J Clin Pract. 2005, 59:1162-1166.

4. Fei Zhou, Ting Yu, Ronghui Du, et al.: Clinical course and risk factors for mortality of adult inpatients with COVID-19 in Wuhan, China: a retrospective cohort study. Lancet. 2020, 395:1054-1062. 10.1016/S01406736(20)30566-3

5. Yuan J, Zou R, Zeng L, et al.: The correlation between viral clearance and biochemical outcomes of 94 COVID-19 infected discharged patients. Inflamm Res. 2020, 69:599-606. 10.1007/s00011-020-01342-0

6. Mao L, Jin H, Wang M, et al.: Neurologic manifestations of hospitalized patients with coronavirus disease 2019 in Wuhan, China. JAMA Neurol. 2020, 77:683-690. 10.1001/jamaneurol.2020.1127

7. Chen N, Zhou M, Dong X, et al.: Epidemiological and clinical characteristics of 99 cases of 2019 novel coronavirus pneumonia in Wuhan, China: a descriptive study. Lancet. 2020, 395:507-513. 10.1016/S01406736(20)30211-7

8. Suwanwongse K, Shabarek N: Rhabdomyolysis as a presentation of 2019 novel coronavirus disease . Cureus. 2020, 12:e7561. 10.7759/cureus.7561

9. Jin M, Tong Q: Rhabdomyolysis as potential late complication associated with COVID-19. Emerg Infect Dis. 2020, 26:1618-1620. 10.3201/eid2607.200445

10. Valente-Acosta B, Moreno-Sanchez F, Fueyo-Rodriguez O, Palomar-Lever A: Rhabdomyolysis as an initial presentation in a patient diagnosed with COVID-19. BMJ Case Rep. 2020, 13:e236719. 10.1136/bcr-2020236719

11. Ronco C, Reis T, Husain-Syed F: Management of acute kidney injury in patients with COVID-19 . Lancet. 2020, 8:738-742. 10.1016/S2213-2600(20)30229-0

12. Su H, Yang M, Wan C, et al.: Renal histopathological analysis of 26 postmortem findings of patients with COVID-19 in China. Kidney Int. 2020, 98:219-227. 10.1016/j.kint.2020.04.003

13. Kellum JA, Aspelin P, Barsoum RS, et al.: Kidney disease: improving global outcomes (KDIGO) acute kidney injury work group. KDIGO clinical practice guideline for acute kidney injury. Kidney Int. 2012, 2:1-138.

14. McMahon GM, Zeng X, Waikar SS: A risk prediction score for kidney failure or mortality in rhabdomyolysis . JAMA Intern Med. 2013, 173:1821-1827. 10.1001/jamainternmed.2013.9774

15. Simpson J, Taylor A, Menon D, Sudhan N, Lavinio A: Rhabdomyolysis: early prognostication of renal failure and other adverse outcomes. Critical Care. 2015, 19:283. 10.1186/cc14363

16. Tian W, Jiang W, Yao J, et al.: Predictors of mortality in hospitalized COVID-19 patients: A systematic review and meta-analysis. J Med Virol. 2020, 92:1875-1883. 10.1002/jmv.26050

17. Bianchetti A, Rozzini R, Guerini F, et al.: Clinical presentation of COVID19 in dementia patients. J Nutr Health Aging. 2020, 24:560-562. 10.1007/s12603-020-1389-1

18. Zhang D, Zhang X, Ma R, et al.: Ultra-fast and onsite interrogation of Severe Acute Respiratory Syndrome Coronavirus 2 (SARS-CoV-2) in environmental specimens via surface enhanced Raman scattering (SERS) [PREPRINT]. Medrxiv. 2020, 10.1101/2020.05.02.20086876

19. Hamming I, Timens W, Bulthuis MLC, Lely AT, Navis GJ, van Goor H: Tissue distribution of ACE2 protein, the functional receptor for SARS coronavirus. A first step in understanding SARS pathogenesis. J Pathol. 2020, 203:631-637. 10.1002/path.1570

20. Ding Y, He L, Zhang Q, et al.: Organ distribution of severe acute respiratory syndrome (SARS) associated coronavirus (SARS-CoV) in SARS patients: implications for pathogenesis and virus transmission pathways. J 


\section{Cureus}

Pathol. 2004, 203:622-630. 10.1002/path.1560 